\title{
Numerical study of large-N phase transition of smeared Wilson loops in 4D pure YM theory
}

\author{
Robert Lohmayer* \\ Rutgers University, Department of Physics and Astronomy, Piscataway, NJ 08854, USA \\ E-mail: lohmayerephysics.rutgers.edu
}

\section{Herbert Neuberger}

Rutgers University, Department of Physics and Astronomy, Piscataway, NJ 08854, USA

E-mail: neuberg@physics.rutgers.edu

\begin{abstract}
In Euclidean four-dimensional $\mathrm{SU}(N)$ pure gauge theory, eigenvalue distributions of Wilson loop parallel transport matrices around closed spacetime curves show non-analytic behavior (a 'large$N$ phase transition') at a critical size of the curve. We focus mainly on an observable composed of traces of the Wilson loop operator in all totally antisymmetric representations, which is regularized with the help of smearing. By studying sequences of square Wilson loops on a hypercubic lattice with standard Wilson action, it is shown that this observable has a nontrivial continuum limit as a function of the physical size of the loop. We furthermore present (preliminary) numerical results confirming that, for large $N$, the $N$ dependence in the critical regime is governed by the universal exponents $1 / 2$ and $3 / 4$ as expected (Burgers universality).
\end{abstract}

XXIX International Symposium on Lattice Field Theory

July 10-16, 2011

Squaw Valley, Lake Tahoe, California

\footnotetext{
* Speaker.
} 


\section{Introduction}

Already in 1981, Durhuus and Olesen [1] discovered that Wilson loops in pure $\mathrm{SU}(N)$ gauge theory in two Euclidean dimensions exhibit a transition from an 'ordered phase' to a 'disordered phase' at large $N$. In two dimensions, the eigenvalue spectrum of the untraced Wilson loop unitary matrix depends only on the area that is enclosed by the (nonselfintersecting) space-time curve defining the loop and a sharp infinite- $N$ phase transition occurs at a critical value of this area. At this point, the gap in the spectrum, that is present for small loops, closes in a non-analytic way.

In Ref. [2], Narayanan and Neuberger conjectured that a similar large- $N$ transition also occurs in three and four dimensions and that all transitions belong to a single universality class. If this is indeed the case, it might be possible to connect the perturbative regime with non-perturbative models in four dimensions using the universal properties of the transition at a critical scale. However, so far the universality conjecture has been confirmed only for the three-dimensional case [3].

In the following, we present a numerical study of the transition and its universal properties in four dimensions using lattice methods.

\section{Main observable}

The Wilson loop matrix associated with a closed spacetime curve $\mathscr{C}$ is defined as usual:

$$
W(\mathscr{C})=\mathscr{P} \exp \left(i \oint_{\mathscr{C}} A_{\mu}(x) d x_{\mu}\right) \in \mathrm{SU}(N)
$$

with path-ordering operator $\mathscr{P}$.

To study the large- $N$ transition in the spectrum of $W$, we focus on

$$
\mathscr{O}_{N}(y, \mathscr{C})=\left\langle\operatorname{det}\left(e^{\frac{y}{2}}+e^{-\frac{y}{2}} W_{f}(\mathscr{C})\right)\right\rangle=\sum_{k=0}^{N} e^{\left(\frac{N}{2}-k\right) y}\left\langle\chi_{k}^{\text {asym }}(W(\mathscr{C}))\right\rangle,
$$

where all totally antisymmetric representations of $W$ enter ( $f$ denotes the fundamental representation and $\chi_{k}^{\text {asym }}$ denotes the character in the $k$-fold totally antisymmetric representation of $\mathrm{SU}(N)$ ). The variable $y$ is defined such that a zero of the determinant at $y=0$ corresponds to an eigenvalueangle $\theta= \pm \pi$ on the complex unit circle. The gap in the eigenvalue spectrum closes at $\theta= \pm \pi$ (cf. Fig. 1 below). Therefore, useful information about the large- $N$ non-analyticity can be obtained by expanding $\mathscr{O}_{N}(y, \mathscr{C})$ in powers of $y$ around $y=0$ (with $\theta_{C P}=0$, only even powers of $y$ enter):

$$
\mathscr{O}_{N}(y, \mathscr{C})=a_{0}(\mathscr{C})+a_{1}(\mathscr{C}) y^{2}+a_{2}(\mathscr{C}) y^{4}+O\left(y^{6}\right) .
$$

In particular, it turns out (see below) that the scaling-invariant ratio

$$
\omega(\mathscr{C})=\frac{a_{0}(\mathscr{C}) a_{2}(\mathscr{C})}{a_{1}(\mathscr{C})^{2}}
$$

provides a valuable signal for the large- $N$ phase transition in the spectrum of $W$. 


\section{Universality class from heat-kernel model}

The heat-kernel probability density (w.r.t. the Haar measure) for an $\mathrm{SU}(N)$ matrix $W$ is given by

$$
\mathscr{P}_{N}^{\mathrm{HK}}(W, t)=\sum_{\text {all irred. } r} d_{r} \chi_{r}(W) e^{-\frac{t}{N} C_{2}(r)}
$$

implying

$$
\left\langle\chi_{r}(W)\right\rangle_{\mathrm{HK}}=d_{r} e^{-\frac{t}{N} C_{2}(r)}
$$

due to character orthogonality. The parameter $t$ can be interpreted as a diffusion time, $d_{r}$ and $C_{2}(r)$ denote the dimension and the quadratic Casimir invariant of the irreducible representation $r$. The above probability density can be realized in a simple multiplicative random matrix model and holds exactly in two-dimensional pure Yang-Mills theory when $t$ is identified with the dimensionless area variable $g^{2} N \mathscr{A}$ (with 't Hooft coupling $g^{2} N$ and $\mathscr{A}$ denoting the area enclosed by the loop). The heat-kernel single eigenvalue-angle distribution is given by [4]

$$
\rho_{N}^{\mathrm{HK}}(\theta, t)=\frac{1}{2 \pi}+\frac{1}{\pi N} \sum_{p=0}^{N-1}(-1)^{p} \sum_{q=0}^{\infty} d(p, q) e^{-\frac{t}{N} C(p, q)} \cos ((p+q+1) \theta)
$$

with $C(p, q)=\frac{1}{2}(p+q+1)\left(N-\frac{p+q+1}{N}+q-p\right)$ and $d(p, q)=\frac{(N+q) !}{p ! q !(N-p-1) !} \frac{1}{p+q+1}$.

An immediate consequence of the group theoretical structure (3.1) is that

$$
\phi_{N}^{\mathrm{HK}}(y, \tau)=-\frac{1}{N} \frac{\partial}{\partial y} \log \mathscr{O}_{N}^{\mathrm{HK}}(y, \tau)=-\frac{1}{N} \frac{\partial}{\partial y} \log \left\langle\operatorname{det}\left(e^{\frac{y}{2}}+e^{-\frac{y}{2}} W_{f}\right)\right\rangle_{\mathrm{HK}}
$$

with $\tau=t(1+1 / N)$ satisfies Burgers' equation [5]

$$
\partial_{\tau} \phi_{N}^{\mathrm{HK}}+\phi_{N}^{\mathrm{HK}} \partial_{y} \phi_{N}^{\mathrm{HK}}=\frac{1}{2 N} \partial_{y}^{2} \phi_{N}^{\mathrm{HK}}
$$

with initial condition $\phi_{N}^{\mathrm{HK}}(y, 0)=-\frac{1}{2} \tanh \frac{y}{2}$. At $N=\infty$, Burgers' equation produces a 'shock-wave' singularity at $y=0$ when $\tau$ reaches the critical value $\tau_{c}=4$, cf. Fig. 1 .
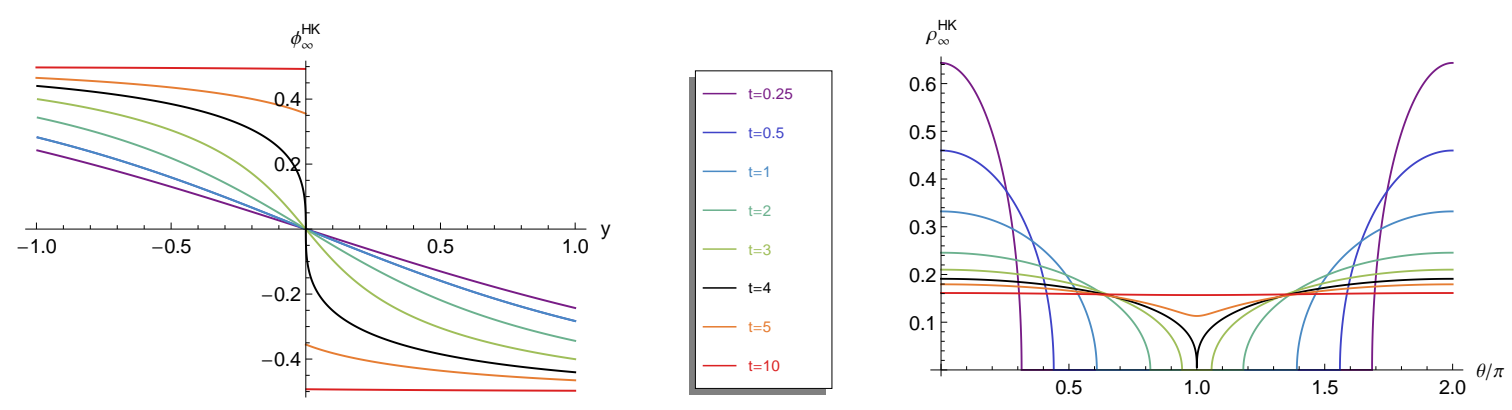

Figure 1: $\phi_{N}^{\mathrm{HK}}$ (left) and heat-kernel single eigenvalue density $\rho_{N}^{\mathrm{HK}}$ (right) in the limit $N \rightarrow \infty$ for various $t$.

From Burgers' equation (at finite $N$ ), we get

$$
\partial_{\tau} \frac{1}{\left.\partial_{y} \phi_{N}^{\mathrm{HK}}\right|_{y=0}}=3 \omega_{N}^{\mathrm{HK}}(\tau)-\frac{1}{2} .
$$


At infinite $N$, the inverse slope of $\phi_{\infty}^{\mathrm{HK}}$ at $y=0$ increases from -4 at $\tau=0$ to 0 at $\tau=4$ since

$$
\omega_{\infty}^{\mathrm{HK}}(\tau)= \begin{cases}1 / 2, & 0 \leq \tau<4, \\ 1 / 6, & \tau>4\end{cases}
$$

resulting in a discontinuous jump in $\phi_{\infty}^{\mathrm{HK}}(y)$ for $\tau>4$ at $y=0$. This discontinuity results in a nonzero single eigenvalue density at $\theta= \pm \pi$ (on the unit circle in the complex plane). The infinite$N$ phase transition (non-analytic behavior with characteristic exponents) in the eigenvalue density of $W$ occurs at the point where the gap in the spectrum disappears (this happens at $\tau=\tau_{c}=4$ ), cf. Fig. 1.

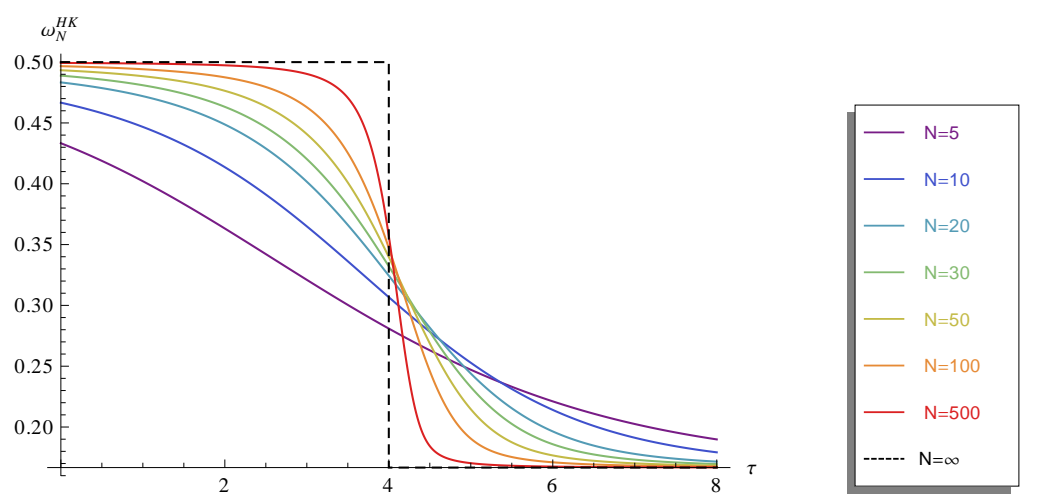

Figure 2: $\omega_{N}^{\mathrm{HK}}(\tau)$ for various $N$.

The singularity is absent for any finite $N$, and very large values of $N$ are needed to see its formation, cf. Fig. 2. From Burgers' equation we obtain:

$$
\begin{aligned}
\left.\lim _{N \rightarrow \infty} N^{-\frac{3}{2}} \frac{a_{1}}{a_{0}}\right|_{\tau=4} & =\frac{1}{8} \sqrt{\frac{3}{2}} \frac{1}{K}, \quad K \equiv \frac{1}{4 \pi} \Gamma^{2}\left(\frac{1}{4}\right) \approx 1.046, \\
\left.\lim _{N \rightarrow \infty} N^{-\frac{3}{2}} \frac{a_{2}}{a_{1}}\right|_{\tau=4} & =\frac{1}{24} \sqrt{\frac{3}{2}} K, \\
\left.\lim _{N \rightarrow \infty} \omega_{N}\right|_{\tau=4} & =\frac{1}{3} K^{2} \\
\left.\lim _{N \rightarrow \infty} N^{-\frac{1}{2}} \frac{d \omega_{N}}{d \tau}\right|_{\tau=4} & =-\frac{1}{6} \sqrt{\frac{3}{2}} K\left(K^{2}-1\right) .
\end{aligned}
$$

Furthermore, the (purely imaginary) roots of $\mathscr{O}_{N}^{\mathrm{HK}}(y, \tau)$ in the critical regime (around $y=0, \tau=4$ ) scale like $N^{-\frac{3}{4}}$.

\section{Numerical results in 4D}

In four dimensions, the Wilson loop operator develops a perimeter divergence (and additional corner divergences if the spacetime curve has kinks). A convenient way of regularization is by smearing (cf. Ref. [6] for details). We deal with the intrinsic UV divergences of the action by using 

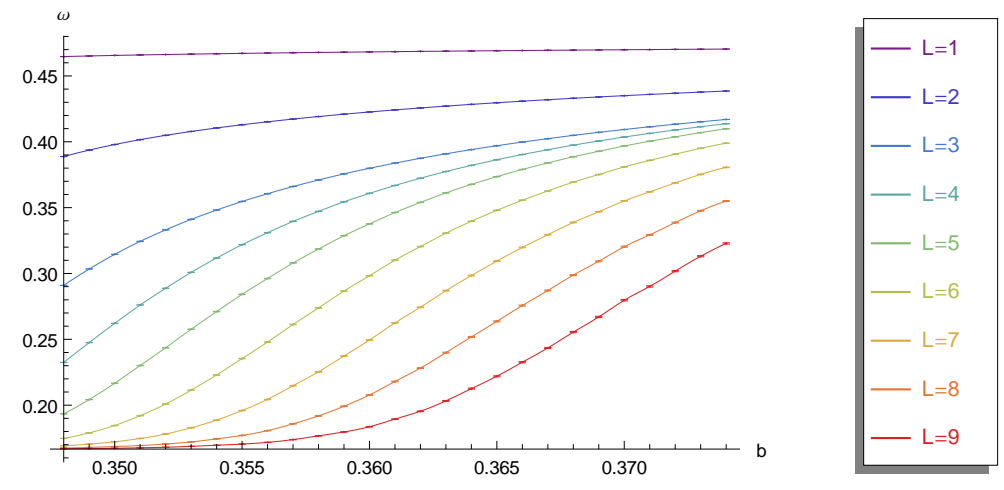

Figure 3: Lattice results for $\omega_{19}(b, L)$ (with cubic spline interpolation between data points).
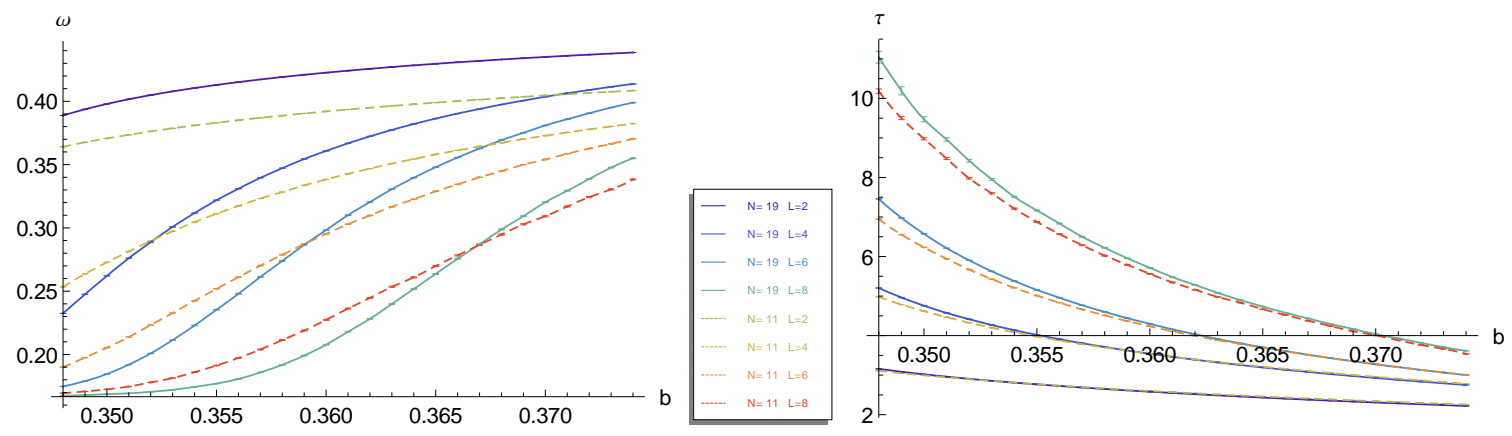

Figure 4: $\omega_{N}(b, L)$ and $\tau_{N}(b, L)$ for $N=19$ (solid) and $N=11$ (dashed) and $L=2,4,6,8$.

a lattice discretization of the theory. To extrapolate to the continuum, we compute sequences of square Wilson loops of sides $1 \leq L \leq 9$ for inverse 't Hooft couplings $0.348 \leq b=\frac{1}{g^{2} N} \leq 0.374$ at $N=19$, using a single plaquette Wilson action on a hypercubic lattice (volumes $12^{4}$ and $14^{4}$ are used to exclude data contaminated by finite-volume effects) and a combination of heat-bath and overrelaxation updates. The amount of smearing $S$ is chosen proportional to the size of the loop, $S=L^{2} / 55$. We then measure $\omega_{N}(b, L)$ (determined from the average characteristic polynomial) as a signal for the large- $N$ phase transition. Large- $N$ scaling is tested with additional runs at $N=11$.

Figure 3 shows our numerical results for $\omega_{19}(b, L)$. Similar to the heat-kernel model, we observe that very large values of $N$ would be needed to allow for a direct observation of the singular large- $N$ behavior in $\omega_{N}$. Therefore, we define an $N$-dependent map $\omega_{N}(b, L) \rightarrow \tau_{N}(b, L)$ through

$$
\omega_{N}(b, L)=\omega_{N}^{\mathrm{HK}}\left(\tau_{N}(b, L)\right) .
$$

The required inversion is unique and the map can be used even though the $r$-dependence of $\left\langle\chi_{r}(W)\right\rangle$ in $4 \mathrm{D}$ differs from exact Casimir scaling. While the formation of the jump in $\omega_{N}$ is slow, $\tau_{N}$ converges rapidly to $\tau_{\infty}$, cf. Fig. 4 . This implies that, to some subleading order in $\frac{1}{N}, \omega_{N}(b, L) \approx$ $\omega_{N}^{\mathrm{HK}}\left(\tau_{\infty}(b, L)\right)$.

This relation can be taken over to the continuum limit, where the two variables $b$ and $L$ get replaced by a single length variable $l=L / L_{c}(b)$, the side of the loop in physical units. To set the 


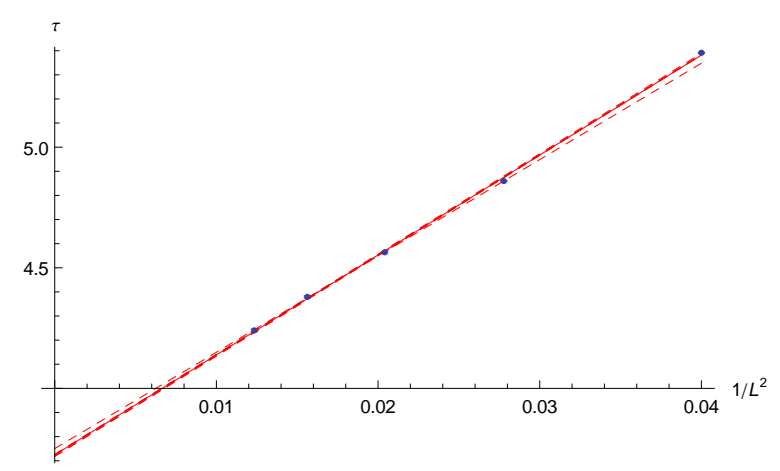

Figure 5: Example for continuum extrapolation at $N=19$ and $\log \left(\frac{L}{L_{c}}\right)=-0.2$.

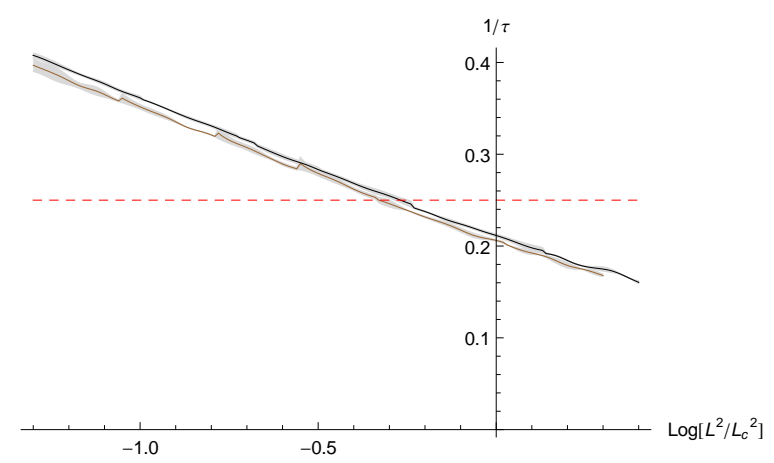

Figure 6: Continuum functions $\tau_{N}(l)^{-1}$ for $N=19$ (black) and $N=11$ (brown). The gray bands show the corresponding error estimates (jackknife), the horizontal dashed line corresponds to the critical value $\tau_{c}=4$.

scale, we use the critical deconfinement temperature $1 / L_{c}(b)$, determined by

$$
L_{c}(b)=0.26\left(\frac{11}{48 \pi^{2} b_{i}(b)}\right)^{\frac{51}{121}} e^{\frac{24 \pi^{2}}{11} b_{i}(b)}, \quad b_{i}(b)=\frac{b}{N}\left\langle\operatorname{Tr} W_{1 \times 1}\right\rangle .
$$

The continuum limit of $\tau_{N}$ is obtained by extrapolating $\tau_{N}(b, L)$ at fixed $l$ to $L \rightarrow \infty$, cf. Fig. 5 .

We observe that $\tau_{N}$ has a nontrivial continuum limit which is a smooth function of the physical loop size $l$ for all $N$, cf. Fig. 6 . This establishes the transition and its universality since we can replace $\mathscr{O}_{N}(y, \mathscr{C})$ by $\mathscr{O}_{N}^{\mathrm{HK}}\left(y, \tau_{\infty}(l)\right)$ in the vicinity of the critical point $(y=0, \tau=4)$ without changing the singular large- $N$ properties. The dependence on $l$ is consistent with asymptotic freedom as $\tau$ modulo a shape-dependent factor can be interpreted as an effective coupling constant.

Additional checks of universality can be obtained from the critical exponent $3 / 2$ of the ratio $a_{0} / a_{1}$ and the $N^{-3 / 4}$-scaling of the level density in the critical region (cf. Fig. 7). Both checks work very well: numerically, we obtain exponents of 1.52 and 0.73 , respectively.

\section{Conclusions}

We have obtained numerical evidence, by extrapolating results of lattice simulations to the 

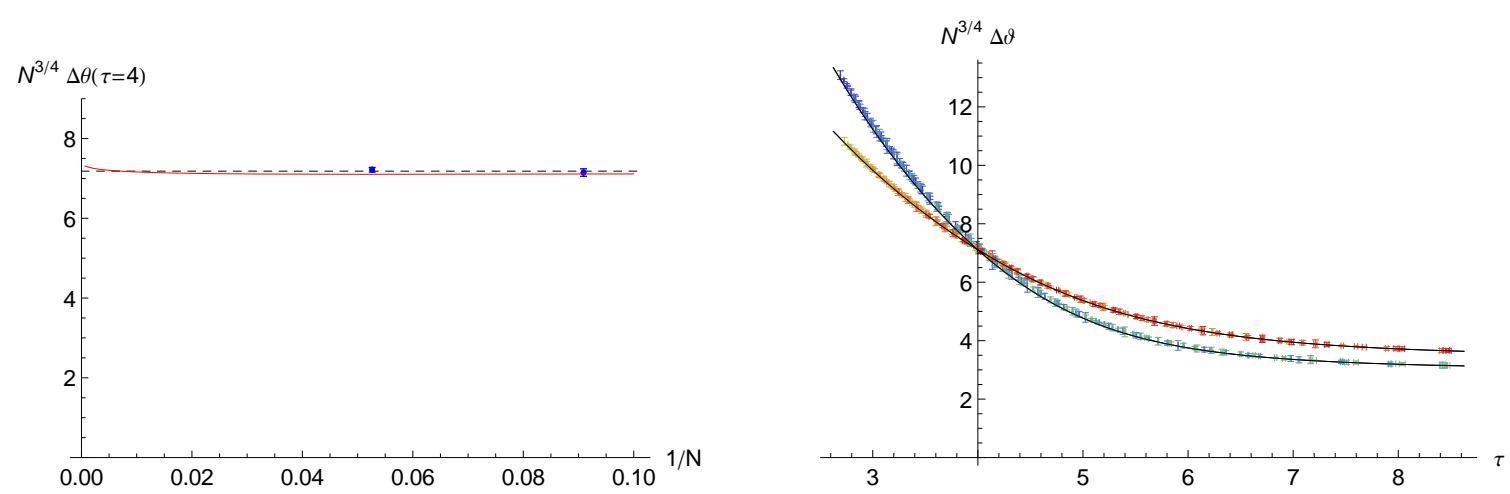

Figure 7: Left: appropriately scaled angular difference $\Delta \theta$ between the two peaks in the single eigenvalue density closest to $\theta=\pi$ at $\tau=4$ as a function of $1 / N$ (blue: data points, red: heat-kernel value).

Right: $N^{3 / 4} \Delta \theta$ as a function of $\tau$ (blue-green points for $N=19$, red-yellow points for $N=11$; black lines show the corresponding heat-kernel functions). At the intersection point (at $\tau=4), \Delta \theta \propto N^{-3 / 4}$.

continuum, that smeared Wilson loops in $4 \mathrm{D}$ continuum pure $\mathrm{SU}(N)$ gauge theory undergo a large$N$ phase transition at a critical loop size. Furthermore, we have confirmed the expected universal properties of the transition. For a complete presentation of our results (including data for larger $N$ ) we refer to Refs. [7, 8].

\section{Acknowledgments}

RL and HN acknowledge partial support by the DOE under grant number DE-FG02-01ER41165. We are grateful to R. Narayanan who was involved in the early stages of this project.

\section{References}

[1] B. Durhuus and P. Olesen, The spectral density for two-dimensional continuum QCD, Nucl. Phys. B184 (1981) 461.

[2] R. Narayanan and H. Neuberger, Infinite N phase transitions in continuum Wilson loop operators, JHEP 03 (2006) 064, [hep-th/ 0601210 ].

[3] R. Narayanan and H. Neuberger, Universality of large N phase transitions in Wilson loop operators in two and three dimensions, JHEP 12 (2007) 066, [arXiv: 0711.4551 ].

[4] R. Lohmayer, H. Neuberger, and T. Wettig, Eigenvalue density of Wilson loops in 2D SU(N) YM, JHEP 05 (2009) 107, [arXiv:0904.4116].

[5] H. Neuberger, Burgers' equation in 2D SU(N) YM, Phys. Lett. B666 (2008) 106-109, [arXiv:0806.0149].

[6] R. Lohmayer and H. Neuberger, Continuous smearing of Wilson Loops, PoS LAT2011 (2011) [arXiv:1110.3522].

[7] R. Lohmayer and H. Neuberger In preparation (2011).

[8] R. Lohmayer and H. Neuberger, Non-analyticity in scale in the planar limit of QCD, arXiv:1109.6683. 